Pacific Journal of Mathematics

ON MCCONNELL'S INEQUALITY FOR FUNCTIONAL O 


\title{
ON MCCONNELL'S INEQUALITY FOR FUNCTIONALS OF SUBHARMONIC FUNCTIONS
}

\author{
AKIHITO UCHIYAMA
}

Recently, McConnell obtained an $L^{p}$ inequality relating the nontangential maximal function of a nonnegative subharmonic function $u$ and an integral expression involving the Laplacian of $u$. His result is imposing a restriction on the range of $p$. In this paper, we show that his inequality holds for all $p \in(0,+\infty)$.

1. Introduction. Let $u(x, t)$ be a nonnegative subharmonic function defined on $R_{+}^{n+1}=\left\{(x, t): x \in R^{n}, t>0\right\}$. (For the definition of subharmonic functions, see Hayman and Kennedy [5] p. 40.) Let $\Delta u$ be the Laplacian of $u$ in the sense of distributions. Then, this is a positive measure on $R_{+}^{n+1}$. Let

$$
\begin{aligned}
& N(x)=\sup \left\{u(y, t):(y, t) \in \Gamma_{1}(x)\right\}, \\
& S(x)=\iint_{(y, t) \in \Gamma_{1}(x)} t^{1-n} \Delta u(y, t),
\end{aligned}
$$

where

$$
\begin{gathered}
\Gamma_{\alpha}(x)=\left\{(y, t) \in R_{+}^{n+1}:|x-y|<\alpha t\right\}, \\
|x|=\left|\left(x_{1}, \ldots, x_{n}\right)\right|=\left(\sum_{i=1}^{n} x_{i}^{2}\right)^{1 / 2} .
\end{gathered}
$$

If $v(x, t)$ is a real harmonic function defined on $R_{+}^{n+1}$ and if

$$
u(x, t)=v(x, t)^{2},
$$

then $u$ is nonnegative and subharmonic. In this case, $N^{1 / 2}$ and $S^{1 / 2}$ turn out to be the usual nontangential maximal function and the usual area integral of $v$, respectively. So, the results of Burkholder and Gundy [1] and C. Fefferman and Stein [3] imply that in case of (1) we have

$$
\|S\|_{L^{p}} \leq c(p, n)\|N\|_{L^{p}}
$$

for all $p \in(0,+\infty)$. (Under the additional assumption $\lim _{t \rightarrow+\infty} v(x, t)$ $=0$, they showed also the converse inequality of (2) with other constants $c(p, n)$. 
Recently, McConnell [7] extended the inequality (2) to general nonnegative subharmonic functions.

THEOREM A. Let $u$ be a nonnegative subharmonic function defined on $R_{+}^{n+1}$. There are constants $c(p, n)<+\infty$, depending only on $p$ and $n$, and a positive constant $p_{0}(n)$, depending only on $n$, such that the inequalities

$$
\|S\|_{L^{p}} \leq c(p, n)\|N\|_{L^{p}}
$$

hold for all $p$ satisfying

$$
0<p<p_{0}(n) \text { or } 1 \leq p<+\infty ;
$$

moreover $p_{0}(1)=1$.

This theorem in the case $n \geq 2$ is imposing an unnatural restriction (4) on the range of $p$. In this paper, we remove (4).

TheOREM 1. Let $u$ be as in Theorem A. Let $0<p<+\infty$. Then, there exist constants $c(p, n)<+\infty$, depending only on $p$ and $n$, such that (3) holds.

The argument in this paper is an extension of that in our paper [8].

2. Preliminaries. First we prepare notation. The Laplacian $\Delta$ and the gradient $\nabla$ in this paper are taken in the sense of distributions. For a measurable subset $E$ of the Euclidean space, let $\chi_{E}$ and $|E|$ be the characteristic function of $E$ and the Lebesgue measure of $E$, respectively. For $x \in R^{n}$ and $E \subset R^{n}$, let $\delta(x, E)$ be the distance of the point $x$ from $E$. Let $\delta(x, \varnothing)=+\infty$.

For $x \in R^{n}, R>1, \alpha>0$, and for $u(x, t)$ in Theorem A let

$$
\begin{aligned}
\varphi(x) & =\max (0,1-|x|), \\
T_{R} & =\left\{(x, t) \in R_{+}^{n+1}:|x|<R, 1 / R<t<R\right\}, \\
N(x ; \alpha) & =\sup \left\{u(y, t):(y, t) \in \Gamma_{\alpha}(x)\right\}, \\
S(x ; \alpha) & =\iint_{(y, t) \in \Gamma_{\alpha}(x)} t^{1-n} \Delta u(y, t), \\
s(x ; \alpha, R) & =\iint_{(y, t) \in R_{+}^{n+1}} \varphi\left(\frac{x-y}{\alpha t}\right) t^{1-n} \Delta u(y, t) \chi_{T_{R}}(y, t) .
\end{aligned}
$$

Note that if $\alpha^{\prime}>\alpha>0$, then

$$
S(x ; \alpha) \leq c\left(\alpha, \alpha^{\prime}, n\right) \lim _{R \rightarrow+\infty} s\left(x ; \alpha^{\prime}, R\right) .
$$


Cubes considered in this paper have sides parallel to the coordinate axes. For a cube $I$, let $l(I)$ and $\alpha I$ be the side length of $I$ and a cube concentric with $I$ satisfying $l(\alpha I)=\alpha l(I)$, respectively. For a cube $I$ in $R^{n}$, let

$$
Q(I)=\left\{(x, t) \in R_{+}^{n+1}: x \in I, t \in(0, l(I))\right\} .
$$

For a nonnegative measure $\mu$ on $R_{+}^{n+1}$ let

$$
\|\mu\|_{c}=\sup _{I} \mu(Q(I)) /|I|
$$

where the supremum is taken over all cubes $I$ in $R^{n}$. If $\|\mu\|_{c}<+\infty$, then $\mu$ is called a Carleson measure.

For the proof of Theorem 1 we need the following.

LeMmA 1. Let $u$ be as in Theorem A. Let $\lambda>0, \alpha>\beta>0$,

$$
\begin{gathered}
\Omega=\left\{x \in R^{n}: N(x ; \alpha) \leq \lambda\right\}, \\
W=\left\{(x, t) \in R_{+}^{n+1}: \delta(x, \Omega)<\beta t\right\} .
\end{gathered}
$$

Then

$$
\left\|t \Delta u \chi_{W}\right\|_{c} \leq C \lambda
$$

where $C$ is a constant depending only on $\alpha, \beta$ and $n$.

LEMMA 2. Let $u$ be as in Theorem A. Let $\lambda>0, R>1, \gamma>1$ and $\alpha>\beta>0$. Then

$$
\begin{aligned}
& \left|\left\{x \in R^{n}: s(x ; \beta, R)>\gamma \lambda, N(x ; \alpha) \leq \lambda\right\}\right| \\
& \quad \leq C e^{-c \gamma}\left|\left\{x \in R^{n}: s(x ; \beta, R)>\lambda\right\}\right|
\end{aligned}
$$

where $C$ and $c$ are positive constants depending only on $\alpha, \beta$ and $n$.

\section{Proof of Lemma 1.}

LEMMA 3. Let $m \geq 2$ be an integer. Let $r>0$,

$$
\begin{aligned}
B & =\left\{X \in R^{m}:|X|<r\right\}, \\
0.5 B & =\left\{X \in R^{m}:|X|<0.5 r\right\} .
\end{aligned}
$$

Let $U(X)$ be a subharmonic function defined on $B$ such that

$$
0 \leq U(X) \leq 1 \text { for all } X \in B
$$


Then

(i) $\Delta U$ in the sense of distributions satisfies

$$
\int_{X \in 0.5 B} \Delta U(X) \leq C r^{m-2},
$$

(ii) $\nabla U$ in the sense of distributions is locally integrable on $B$ and satisfies

$$
\int_{X \in 0.5 B}|\nabla U(X)| \leq C r^{m-1}
$$

where $C$ is a constant depending only on $m$.

Proof. We may assume $r=1$. Let $G(X, Y)$ be the Green function of $B=\left\{X \in R^{m}:|X|<1\right\}$. Namely, for $(X, Y) \in(B \times B) \backslash\{(X, X): X \in$ $B\}$, let

$$
G(X, Y)= \begin{cases}|X-Y|^{2-m}-|| Y|X-Y /| Y||^{2-m}, & Y \neq 0 \\ |X|^{2-m}-1, & Y=0\end{cases}
$$

if $m \geq 3$ and let

$$
G(X, Y)= \begin{cases}\log \frac{\| Y|X-Y /| Y||}{|X-Y|}, & Y \neq 0 \\ \log \frac{1}{|X|}, & Y=0\end{cases}
$$

if $m=2$. For $Y \in B$ let

$$
V(Y)=\frac{1}{\sigma_{m}} \int_{X \in 0.6 B} G(X, Y) \Delta U(X),
$$

where

$$
\sigma_{m}=\frac{2 \pi^{m / 2} \max (1, m-2)}{\Gamma(m / 2)} .
$$

Since $U+V$ is nonnegative on $B$, harmonic on $0.6 B$, subharmonic on $B$ and

$$
\lim _{\varepsilon \rightarrow+0} \sup \left\{V(Y): Y \in R^{m},|Y|=1-\varepsilon\right\}=0,
$$

we have

$$
0 \leq U(Y)+V(Y) \leq 1 \text { on } B
$$




$$
|\nabla(U+V)(Y)| \leq C \text { on } 0.5 B .
$$

Therefore,

$$
\begin{aligned}
c \int_{X \in 0.6 B} \Delta U(X) & \leq \int_{X \in 0.6 B} G(X, 0) \Delta U(X) \\
& =\sigma_{m} V(0) \leq \sigma_{m}(U(0)+V(0)) \leq \sigma_{m}
\end{aligned}
$$

by (10) and

$$
\begin{aligned}
\int_{Y \in B}|\nabla V(Y)| & \leq \frac{1}{\sigma_{m}} \int_{X \in 0.6 B} \Delta U(X) \int_{Y \in B}\left|\nabla_{Y} G(X, Y)\right| \\
& \leq C \int_{X \in 0.6 B} \Delta U(X) \leq C
\end{aligned}
$$

by (12), where $c>0$ and $C<+\infty$ depend only on $m$. So, (i) follows from (12) and (ii) follows from (11) and (13).

Now, we begin the proof of Lemma 1. We may assume

$$
\lambda=1
$$

and $\Omega \neq \varnothing$. Let a cube $I$ be given. It is enough to show

$$
\iint_{(x, t) \in Q(I) \cap W} t \Delta u(x, t) \leq C|I| .
$$

Let $\varepsilon>0$ be a constant such that

$$
\begin{gathered}
\varepsilon<\min \left(\frac{\beta}{n^{1 / 2}}, \frac{1}{n^{1 / 2}}\right), \\
\frac{(1+\varepsilon)(\beta+\varepsilon)+2 n \varepsilon}{1-n^{1 / 2} \varepsilon}<\alpha .
\end{gathered}
$$

For $\eta>0, x \in R^{n}$ and $t>0$, let

$$
\psi_{\eta}(x)=\max (\delta(x, \Omega), \delta(x, I), \eta),
$$

(19) $\varphi_{\eta}(x, t)= \begin{cases}1 & \text { if } \frac{1}{\beta} \psi_{\eta}(x)<t, \\ \frac{\beta\left((\beta+\varepsilon) t-\psi_{\eta}(x)\right)}{\varepsilon \psi_{\eta}(x)} & \text { if } \frac{1}{\beta+\varepsilon} \psi_{\eta}(x)<t \leq \frac{1}{\beta} \psi_{\eta}(x), \\ 0 & \text { if } t \leq \frac{1}{\beta+\varepsilon} \psi_{\eta}(x),\end{cases}$ 
(20)

$$
h(t)= \begin{cases}0 & \text { if }(1+\varepsilon) l(I)<t, \\ \frac{(1+\varepsilon) l(I)-t}{\varepsilon l(I)} & \text { if } l(I)<t \leq(1+\varepsilon) l(I), \\ 1 & \text { if } t \leq l(I),\end{cases}
$$

$$
V_{\eta}(x, t)=\varphi_{\eta}(x, t) h(t) .
$$

Let $R_{+}^{n+1}=\bigcup_{k=1}^{\infty} Q_{k}$ be the Whitney decomposition of $R_{+}^{n+1}$ such that $\left\{Q_{k}\right\}_{k=1}^{\infty}$ are dyadic cubes in $R_{+}^{n+1}$ with disjoint interiors,

$$
\frac{1}{\varepsilon} l\left(Q_{k}\right) \leq\left(\text { distance between } Q_{k} \text { and } \partial R_{+}^{n+1}\right) \leq \frac{4}{\varepsilon} l\left(Q_{k}\right) \text {, }
$$

(This collection $\left\{Q_{k}\right\}$ can be obtained by taking all the maximal cubes among the closed dyadic cubes in $R_{+}^{n+1}$ that satisfy (23).) Let $\left\{Q_{k(j)}\right\}_{j=1}^{N}$ be the subcollection of $\left\{Q_{k}\right\}$ such that

$$
Q_{k(j)} \cap \operatorname{supp} \nabla V_{\eta} \neq \varnothing .
$$

In the following part of this section, the letter $C$ denotes various positive constants depending only on $\alpha, \beta, \varepsilon$ and $n$.

First we accept the following (25)-(30) temporarily;

$$
\begin{gathered}
\left|\nabla_{\eta}(x, t)\right| \leq \frac{C}{t}, \\
\operatorname{supp} \nabla V_{\eta} \subset \bigcup_{j=1}^{N} Q_{k(j)}, \\
\iint_{\operatorname{supp} \nabla V_{\eta}} \frac{1}{t} d x d t \leq C|I|, \\
\bigcup_{j=1}^{N} 2 n^{1 / 2} Q_{k(j)} \subset\left\{(x, t) \in R_{+}^{n+1}: u(x, t) \leq 1\right\}, \\
\sum_{j=1}^{N}\left(l\left(Q_{k(j)}\right)\right)^{n} \leq C|I|,
\end{gathered}
$$

the left-hand side of $(15)$

$$
\leq \lim _{\eta \rightarrow+0} \iint_{(x, t) \in R_{+}^{n+1}} t V_{\eta}(x, t) \Delta u(x, t) .
$$

Then, (28) and Lemma 3 (ii) imply

$$
\iint_{(x, t) \in Q_{k(j)}}|\nabla u(x, t)| \leq C\left(l\left(Q_{k(j)}\right)\right)^{n} .
$$


Thus,

(32)

$$
\begin{aligned}
\left|\iint_{(x, t) \in R_{+}^{n+1}} t V_{\eta}(x, t) \frac{\partial^{2} u}{\partial x_{i}^{2}}(x, t)\right|=\left|-\iint t \frac{\partial V_{\eta}}{\partial x_{i}} \frac{\partial u}{\partial x_{i}}\right| & \\
\leq C \iint_{\text {supp } \nabla V_{\eta}}\left|\frac{\partial u}{\partial x_{i}}\right| & \text { by (25) } \\
\leq C \sum_{j=1}^{N} \iint_{Q_{k(j)}}|\nabla u| & \text { by (26) } \\
\leq C \sum\left(l\left(Q_{k(j)}\right)\right)^{n} & \text { by (31) } \\
\leq C|I| & \text { by (29) }
\end{aligned}
$$

and

$$
\begin{aligned}
\left|\iint_{(x, t) \in R_{+}^{n+1}} t V_{\eta} \frac{\partial^{2} u}{\partial t^{2}}\right| & =\left|\iint\left(-V_{\eta}-t \frac{\partial V_{\eta}}{\partial t}\right) \frac{\partial u}{\partial t}\right| \\
& =\left|\iint \frac{\partial V_{\eta}}{\partial t} u-\iint t \frac{\partial V_{\eta}}{\partial t} \frac{\partial u}{\partial t}\right| \\
& =|(33)-(34)|,
\end{aligned}
$$

where

$$
\begin{aligned}
|(33)| & \leq \iint_{\operatorname{supp} \nabla V_{\eta}} \frac{C}{t} u(x, t) d x d t & & \text { by }(25) \\
& \leq \iint_{\operatorname{supp} \nabla V_{\eta}} \frac{C}{t} d x d t & & \text { by }(28) \text { and }(26) \\
& \leq C|I| & & \text { by }(27)
\end{aligned}
$$

and where

$$
|(34)| \leq C|I|
$$

follows from the same argument as (32). Thus we get

$$
\iint t V_{\eta}(x, t) \Delta u(x, t) \leq C|I|
$$

which combined with (30) implies (15).

Next, we prove (25)-(30). (25)-(26) are clear. (30) follows from

$$
\begin{aligned}
\{(x, t) \in & \left.R_{+}^{n+1}: V_{\eta}(x, t)=1\right\} \\
& \supset\left\{(x, t) \in R_{+}^{n+1}: \frac{1}{\beta} \psi_{\eta}(x) \leq t \leq l(I)\right\} \\
& \supset Q(I) \cap W \cap\left\{(x, t) \in R_{+}^{n+1}: t>\eta\right\} .
\end{aligned}
$$


Proof of (28). Since

$$
\begin{aligned}
\operatorname{supp} \nabla V_{\eta} & \subset \operatorname{supp} V_{\eta} \\
& \subset\left\{(x, t) \in R_{+}^{n+1}: \frac{1}{\beta+\varepsilon} \psi_{\eta}(x)<t\right\} \\
& \subset\left\{(x, t) \in R_{+}^{n+1}: \frac{1}{\beta+\varepsilon} \delta(x, \Omega)<t\right\} \\
& =\bigcup_{x \in \Omega} \Gamma_{\beta+\varepsilon}(x),
\end{aligned}
$$

for each $Q_{k(j)}$ there exists an $x \in \Omega$ such that

$$
Q_{k(j)} \cap \Gamma_{\beta+\varepsilon}(x) \neq \varnothing,
$$

which combined with (17) and (23) implies

$$
2 n^{1 / 2} Q_{k(j)} \subset \Gamma_{\alpha}(x) .
$$

Therefore,

$$
\begin{aligned}
\bigcup_{j=1}^{N} 2 n^{1 / 2} Q_{k(j)} & \subset \bigcup_{x \in \Omega} \Gamma_{\alpha}(x) \\
& \subset\left\{(x, t) \in R_{+}^{n+1}: u(x, t) \leq 1\right\} \text { by }(14) .
\end{aligned}
$$

Proof of (27). Let

$$
\tilde{I}=(1+2(\beta+\varepsilon)(1+\varepsilon)) I .
$$

Then

(35) $\operatorname{supp} \nabla V_{\eta} \subset \operatorname{supp} V_{\eta}$

$$
\begin{aligned}
& \subset\left\{(x, t) \in R_{+}^{n+1}: \frac{1}{\beta+\varepsilon} \delta(x, I) \leq t \leq(1+\varepsilon) l(I)\right\} \\
& \subset Q(\tilde{I}) .
\end{aligned}
$$

Let

$$
\begin{aligned}
& S_{1}=\left\{(x, t) \in R_{+}^{n+1}: \frac{1}{\beta+\varepsilon} \psi_{\eta}(x) \leq t \leq \frac{1}{\beta} \psi_{\eta}(x)\right\}, \\
& S_{2}=\left\{(x, t) \in R_{+}^{n+1}: l(I) \leq t \leq(1+\varepsilon) l(I)\right\} .
\end{aligned}
$$

Then, by (19)-(21) and (35) we have

$$
\operatorname{supp} \nabla V_{\eta} \subset\left(S_{1} \cup S_{2}\right) \cap Q(\tilde{I}),
$$

which combined with (25) implies (27). 
Proof of (29). Let

$$
\begin{aligned}
\tilde{S}_{1}= & \left\{(x, t) \in R_{+}^{n+1}:\right. \\
& \left.\frac{1}{(1+\varepsilon)(\beta+\varepsilon)+n^{1 / 2} \varepsilon} \psi_{\eta}(x) \leq t \leq \frac{1+\varepsilon}{\beta-n^{1 / 2} \varepsilon} \psi_{\eta}(x)\right\}, \\
\tilde{S}_{2}= & \left\{(x, t) \in R_{+}^{n+1}: \frac{1}{1+\varepsilon} l(I) \leq t \leq(1+\varepsilon)^{2} l(I)\right\} .
\end{aligned}
$$

It follows from (23) that

$$
\text { if } Q_{k} \cap S_{i} \neq \varnothing, \text { then } Q_{k} \subset \tilde{S}_{i},
$$

for $i=1,2$, respectively. (The case $i=2$ is clear. The proof for the case $i=1$ needs the Lipschitz continuity of $\psi_{\eta}$.) Thus, (36) and (24) imply

$$
\bigcup_{j=1}^{N} Q_{k(j)} \subset \tilde{S}_{1} \cup \tilde{S}_{2} .
$$

On the other hand, (23)-(24) and (35) imply

$$
\bigcup_{j=1}^{N} Q_{k(j)} \subset Q((1+2 \varepsilon) \tilde{I}) \text {. }
$$

For $(x, t) \in R_{+}^{n+1}$ let $P(x, t)=x$. Then, by (22)-(23), (37) and by the geometrical properties of $\tilde{S}_{1}$ and $\tilde{S}_{2}$, we have

$$
\left\|\sum_{j=1}^{N} \chi_{P\left(Q_{k(J)}\right)}(x)\right\|_{L^{\infty}\left(R^{n}\right)} \leq C,
$$

which combined with (38) implies

$$
\begin{aligned}
\sum_{j=1}^{N}\left(l\left(Q_{k(j)}\right)\right)^{n} & =\sum_{j}\left|P\left(Q_{k(j)}\right)\right| \\
& \leq C\left|\bigcup_{j} P\left(Q_{k(j)}\right)\right| \leq C|I| .
\end{aligned}
$$

4. Proof of Lemma 2. In the rest of this paper, the letter $C$ denotes various positive constants depending only on $\alpha, \beta$ and $n$.

We continue to assume (14).

Let

$$
\mathscr{S}(x)=\iint_{(y, t) \in R_{+}^{n+1}} \varphi\left(\frac{x-y}{\beta t}\right) t^{1-n} \Delta u(y, t) \chi_{W \cap T_{R}}(y, t) .
$$


Note that

$$
\begin{array}{lc}
\mathscr{S}(x)=s(x ; \beta, R) & \text { on } \Omega, \\
\mathscr{S}(x) \leq s(x ; \beta, R) & \text { on } R^{n} .
\end{array}
$$

Since $\mathscr{S}(x)<+\infty$ and since $\mathscr{S}(x)$ is the balayage of the Carleson measure $t \Delta u \chi_{W \cap T_{R}}$ with respect to the kernel $\varphi(x)$, which has a compact support and which belongs to the Lipschitz class, a well-known estimate of the BMO-norm in terms of the norm of Carleson measure gives us

$$
\|\mathscr{S}\|_{\mathrm{BMO}} \leq C\left\|t \Delta u \chi_{W \cap T_{R}}\right\|_{c}
$$

which combined with Lemma 1 and (14) implies

$$
\|\mathscr{S}\|_{\mathrm{BMO}} \leq C \text {. }
$$

Thus, the left-hand side of (9) with (14)

$$
\begin{aligned}
& =|\{\mathscr{S}(x)>\gamma, N(x ; \alpha) \leq 1\}| \\
& \leq|\{\mathscr{S}(x)>\gamma\}| \leq{ }^{(*)} C e^{-c \gamma}|\{\mathscr{S}(x)>1\}| \\
& \leq C e^{-c \gamma}|\{s(x ; \beta, R)>1\}| \\
& =\text { the right-hand side of (9) with (14), }
\end{aligned}
$$

where the inequality $(*)$ follows from (41) and from an easy modification of the result of John-Nirenberg [6]. (See Lemma 2.1 of [8] for details.)

5. Proof of Theorem 1. Let $\beta^{\prime}=(\alpha+\beta) / 2$. Applying Lemma 2 with $\beta$ replaced by $\beta^{\prime}$ gives us

$$
\begin{aligned}
& \left|\left\{s\left(x ; \beta^{\prime}, R\right)>\gamma \lambda\right\}\right| \\
& \quad \leq\left|\left\{s\left(x ; \beta^{\prime}, R\right)>\gamma \lambda, N(x ; \alpha) \leq \lambda\right\}\right|+|\{N(x ; \alpha)>\lambda\}| \\
& \quad \leq C e^{-c \gamma}\left|\left\{s\left(x ; \beta^{\prime}, R\right)>\lambda\right\}\right|+|\{N(x ; \alpha)>\lambda\}| .
\end{aligned}
$$

Thus,

$$
\begin{aligned}
& \gamma^{-p}\left\|s\left(\cdot ; \beta^{\prime}, R\right)\right\|_{L^{p}}^{p}=p \int_{0}^{+\infty} \lambda^{p-1}\left|\left\{s\left(x ; \beta^{\prime}, R\right)>\gamma \lambda\right\}\right| d \lambda \\
& \quad \leq p \int_{0}^{+\infty} \lambda^{p-1}\left(C e^{-c \gamma}\left|\left\{s\left(x ; \beta^{\prime}, R\right)>\lambda\right\}\right|+|\{N(x ; \alpha)>\lambda\}|\right) d \lambda \\
& \quad=C e^{-c \gamma}\left\|s\left(\cdot ; \beta^{\prime}, R\right)\right\|_{L^{p}}^{p}+\|N(\cdot ; \alpha)\|_{L^{p}}^{p} .
\end{aligned}
$$

Since $\left\|s\left(\cdot ; \beta^{\prime}, R\right)\right\|_{L^{p}}<+\infty$, the above inequality with sufficiently large $\gamma$ implies

$$
2^{-1} \gamma^{-p}\left\|s\left(\cdot ; \beta^{\prime}, R\right)\right\|_{L^{p}}^{p} \leq\|N(\cdot ; \alpha)\|_{L^{p}}^{p}
$$


Letting $R \rightarrow+\infty$ and recalling (5), we get

$$
\|S(\cdot ; \beta)\|_{L^{p}} \leq C(\alpha, \beta, p, n)\|N(\cdot ; \alpha)\|_{L^{p}} .
$$

On the other hand, the argument of [3], p. 166, Lemma 1 shows

$$
c(\alpha, p, n)\|N\|_{L^{p}} \leq\|N(\cdot ; \alpha)\|_{L^{p}} \leq C(\alpha, p, n)\|N\|_{L^{p}} .
$$

The argument of [2], p. 19, Theorem 3.4 and that of [7], p. 296, Lemma 3.3 show

$$
c(\beta, p, n)\|S\|_{L^{p}} \leq\|S(\cdot ; \beta)\|_{L^{p}} \leq C(\beta, p, n)\|S\|_{L^{p}},
$$

where

$$
\begin{aligned}
& 0<c(\alpha, p, n), \quad c(\beta, p, n) \quad \text { and } \\
& C(\alpha, p, n), \quad C(\beta, p, n)<+\infty
\end{aligned}
$$

Therefore, Theorem 1 follows from (42)-(44).

\section{REFERENCES}

[1] D. L. Burkholder and R. F. Gundy, Distribution function inequalities for the area integral, Studia Math., 44 (1972), 527-544.

[2] A. Calderon and A. Torchinsky, Parabolic maximal functions associated with a distribution, Advances in Math., 16 (1975), 1-63.

[3] C. Fefferman and E. M. Stein, $H^{p}$ spaces of several variables, Acta Math., 129 (1972), 137-193.

[4] R. Fefferman, R. F. Gundy, M. Silverstein and E. M. Stein, Inequalities for ratios of functionals of harmonic functions, Proc. Nat. Acad. Sci. USA, 79 (1982), 7958-7960.

[5] W. K. Hayman and P. B. Kennedy, Subharmonic Functions, Academic Press 1976.

[6] F. John and L. Nirenberg, On functions of bounded mean oscillation, Comm. Pure Appl. Math., 14 (1961), 415-426.

[7] T. R. McConnell, Area integrals and subharmonic functions, Indiana Univ. Math. J., 33 (1984), 289-303.

[8] T. Murai and A. Uchiyama, Good $\lambda$ inequalities for the area integral and the nontangential maximal function, to appear in Studia Math., 83 (1986), 47-58.

[9] E. M. Stein, Singular integrals and differentiability properties of functions, Princeton, 1970.

Received March 14, 1986. Supported in part by Grant-in-Aid for Scientific Research No. 60740064, Japan.

TOHOKU UNIVERSITY

SENDAI, JAPAN 



\title{
PACIFIC JOURNAL OF MATHEMATICS EDITORS
}

\author{
V. S. VARADARAJAN \\ (Managing Editor) \\ University of California \\ Los Angeles, CA 90024 \\ HERBERT Clemens \\ University of Utah \\ Salt Lake City, UT 84112 \\ R. FINN \\ Stanford University \\ Stanford, CA 94305
}

\author{
HERMANN FLASCHKA \\ University of Arizona \\ Tucson, AZ 85721 \\ RAMESH A. GANGOLLI \\ University of Washington \\ Seattle, WA 98195 \\ VAUGHAN F. R. JONES \\ University of California \\ Berkeley, CA 94720 \\ ROBION KIRBY \\ University of California \\ Berkeley, CA 94720
}

\author{
C. C. Moore \\ University of California \\ Berkeley, CA 94720
}

H. SAMELSON

Stanford University

Stanford, CA 94305

HAROLD STARK

University of California, San Diego

La Jolla, CA 92093

\section{ASSOCIATE EDITORS}

\author{
R. ARENS \\ E. F. BECKENBACH \\ B. H. NEUMANN \\ F. WOLF \\ K. YOSHIDA \\ (1906-1982)

\section{SUPPORTING INSTITUTIONS} \\ UNIVERSITY OF ARIZONA \\ UNIVERSITY OF BRITISH COLUMBIA \\ CALIFORNIA INSTITUTE OF TECHNOLOGY \\ UNIVERSITY OF CALIFORNIA \\ MONTANA STATE UNIVERSITY \\ UNIVERSITY OF NEVADA, RENO \\ NEW MEXICO STATE UNIVERSITY \\ OREGON STATE UNIVERSITY

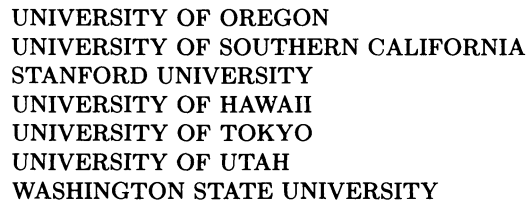

The Supporting Institutions listed above contribute to the cost of publication of this Journal, but they are not owners or publishers and have no responsibility for its content or policies.

Mathematical papers intended for publication in the Pacific Journal of Mathematics should be in typed form or offset-reproduced (not dittoed), double spaced with large margins. Please do not use built up fractions in the text of the manuscript. However, you may use them in the displayed equations. Underline Greek letters in red, German in green, and script in blue. The first paragraph must be capable of being used separately as a synopsis of the entire paper. In particular it should contain no bibliographic references. Please propose a heading for the odd numbered pages of less than 35 characters. Manuscripts, in triplicate, may be sent to any one of the editors. Please classify according to the scheme of Math. Reviews, Index to Vol. 39. Supply name and address of author to whom proofs should be sent. All other communications should be addressed to the managing editor, or Elaine Barth, University of California, Los Angeles, California 90024.

There are page-charges associated with articles appearing in the Pacific Journal of Mathematics. These charges are expected to be paid by the author's University, Government Agency or Company. If the author or authors do not have access to such Institutional support these charges are waived. Single authors will receive 50 free reprints; joint authors will receive a total of $\mathbf{1 0 0}$ free reprints. Additional copies may be obtained at cost in multiples of 50 .

The Pacific Journal of Mathematics is issued monthly as of January 1966. Regular subscription rate: $\$ 190.00$ a year (5 Vols., 10 issues). Special rate: $\$ 95.00$ a year to individual members of supporting institutions.

Subscriptions, orders for numbers issued in the last three calendar years, and changes of address should be sent to Pacific Journal of Mathematics, P.O. Box 969, Carmel Valley, CA 93924, U.S.A. Old back numbers obtainable from Kraus Periodicals Co., Route 100, Millwood, NY 10546.

The Pacific Journal of Mathematics at P.O. Box 969, Carmel Valley, CA 93924 (ISSN 0030-8730) publishes 5 volumes per year. Application to mail at Second-class postage rates is pending at Carmel Valley, California, and additional mailing offices. Postmaster: send address changes to Pacific Journal of Mathematics, P.O. Box 969, Carmel Valley, CA 93924.

PUBLISHED BY PACIFIC JOURNAL OF MATHEMATICS, A NON-PROFIT CORPORATION Copyright (C) 1987 by Pacific Journal of Mathematics 


\section{Pacific Journal of Mathematics}

Vol. 128 , No. $2 \quad$ April, 1987

Pierre Barrucand, John Harold Loxton and Hugh C. Williams, Some

explicit upper bounds on the class number and regulator of a cubic field

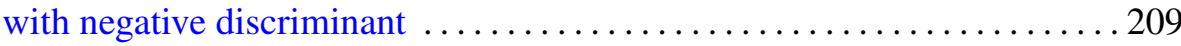

Thomas Ashland Chapman, Piecewise linear fibrations $\ldots \ldots \ldots \ldots \ldots 223$

Yves Félix and Jean-Claude Thomas, Extended Adams-Hilton's

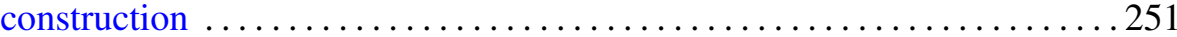

Robert Fitzgerald, Derivation algebras of finitely generated Witt rings . . . 265

K. Gopalsamy, Oscillatory properties of systems of first order linear delay differential inequalities ................................ 299

John P. Holmes, One parameter subsemigroups in locally complete

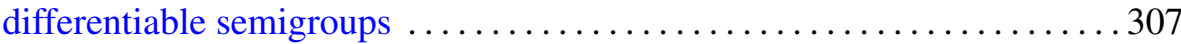

Douglas Murray Pickrell, Decomposition of regular representations for

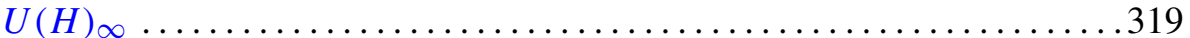

Victoria Powers, Characterizing reduced Witt rings of higher level

Parameswaran Sankaran and Peter Zvengrowski, Stable parallelizability

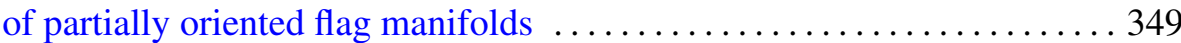

Johan Tysk, Eigenvalue estimates with applications to minimal surfaces . . . 361 Akihito Uchiyama, On McConnell's inequality for functionals of subharmonic functions

Minato Yasuo, Bott maps and the complex projective plane: a construction

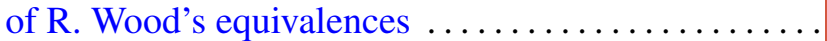

James Juei-Chin Yeh, Uniqueness of strong solutions to stochastic differential equations in the plane with deterministic boundary process 